\title{
Editorial: Mechanisms of Cell Adhesion in Hematopoietic Stem Cells
}

\author{
Shinobu Matsuura ${ }^{1 *}$, Alessandra Balduini ${ }^{2,3}$ and Maurizio Onisto ${ }^{4}$ \\ ${ }^{1}$ Whitaker Cardiovascular Institute, Cardiovascular Section, Department of Medicine, Boston University School of Medicine, \\ Boston, MA, United States, ${ }^{2}$ Department of Molecular Medicine, University of Pavia, Pavia, Italy, ${ }^{3}$ Department of Biomedical \\ Engineering, Tufts School of Engineering, Tufts University, Medford, MA, United States, ${ }^{4}$ Department of Biomedical Sciences, \\ School of Medicine and Surgery, University of Padua, Padua, Italy
}

Keywords: hematopoietic stem cell, stem cell niche, adhesion, extracellular matrix, laminin, acute myeloid leukemia, leukemia stem cell, tetraspanin

\section{Editorial on the Research Topic}

\section{Mechanisms of Cell Adhesion in Hematopoietic Stem Cells}

Four decades after Lord et al. (1975) and Schofield (1978) proposed the concept of a specialized microenvironment in bone marrow in support of hematopoietic stem cell function (Scadden, 2014), the field is more active and vibrant than ever. Seminal works contributed to define the elements that constitute the hematopoietic stem cell niche, or, perhaps, niches: accumulated evidence suggests that the location of the bone marrow hematopoietic niche is perivascular, with a possible contribution of the bone endosteal space. Supporting structures in those environments include cells (endothelial cells, perivascular mesenchymal stromal cells, megakaryocytes, sympathetic neurons and Schwann cells, etc.), bone and bone lining cells (osteoblast, osteoclast) and extracellular matrix (fibronectin, collagen, osteopontin, hyaluronan, tenascin, etc.). As the physical components of the niche are defined, new questions come to the fore: what the molecular mechanisms involved in HSC adhesion are; whether, and by what mechanisms, adhesion may control HSC stemness, proliferation and differentiation; how adhesion mechanisms in HSC differ from those in more mature progenitors or terminally differentiated cells; what disturbances in adhesion are observed in disease states; and whether we can manipulate stem cell function through manipulation of adhesion. In this topic, we present a lineup of exciting and impactful publications responding to these questions. Approaching the questions from different angles and perspectives, the original publications and reviews presented here provide novel data and insightful information that will inspire the reader and will lead the way to future discoveries in the field.

Laminin is an extracellular matrix adhesion-protein present in the basement membrane of most vascular structures in bone marrow, including sinusoids and arteries (Coutu et al., 2017). Godavarty et al., using human $\mathrm{CD}_{3} 4^{+}$cells, examine the role of two novel laminin receptors, $\alpha 7 \beta 1$ and basal cell adhesion molecule/Lutheran (BCAM/Lu), in hematopoietic stem cell biology. BCAM/Lu, which interacts exclusively with laminin has so far only been detected in the erythroid lineage (De Grandis et al., 2013), but not on early hematopoietic stem and progenitor cells (HSPC).

Acute myeloid leukemia (AML) is a heterogeneous, complex, and deadly disease. Therapy is still highly reliant on intensive chemotherapy, which is efficient in reducing the bulk of leukemic cells, but is not effective at eliminating the leukemia stem cells (LSC). The presence of residual LSC is believed to be responsible for relapse and chemotherapy resistance; hence, the urgent need for therapies that 
can specifically target the LSC. Mirroring their healthy counterpart, LSC are nested in specialized niches which support their stemness, competition advantage, and acquisition of resistance to therapy. Villatoro et al. provide a comprehensive review of the most recent advances in the understanding of LSC niches, and provide an overview of clinical trials aiming at targeting these processes for AML treatment. Erbani et al. report on the important novel discovery of the role of CD162, receptor of E-selectin, as a key AML cell surface receptor involved in AML progression, bone marrow retention and chemoresistance. Their findings highlight specific blockade of AML cell surface CD162 as a potential novel niche-based strategy to improve the efficacy of AML therapy.

As is usually the case in science, embarking on our journey of discovery has left us pleasantly surprised by the direction it is taking us. Future directions of the field are provided by two excellent reviews in this topic. Kulkarni and Kale provide a detailed review of reported adhesion molecules in hematopoietic stem cells, and discuss physiological cues involved in their regulation, with special focus on extracellular

\section{REFERENCES}

Coutu, D. L., Kokkaliaris, K. D., Kunz, L., and Schroeder, T. (2017). Threedimensional Map of Nonhematopoietic Bone and Bone-Marrow Cells and Molecules. Nat. Biotechnol. 35 (12), 1202-1210. doi:10.1038/nbt.4006

De Grandis, M., Cambot, M., Wautier, M.-P., Cassinat, B., Chomienne, C., Colin, Y., et al. (2013). JAK2V617F Activates Lu/BCAM-Mediated Red Cell Adhesion in Polycythemia Vera through an EpoR-independent Rap1/Akt Pathway. Blood 121 (4), 658-665. doi:10.1182/blood-2012-07-440487

Lord, B., Testa, N., and Hendry, J. (1975). The Relative Spatial Distributions of CFUs and CFUc in the normal Mouse Femur. Blood 46 (1), 65-72. doi:10.1182/ blood.v46.1.65.bloodjournal46165

Scadden, D. T. (2014). Nice Neighborhood: Emerging Concepts of the Stem Cell Niche. Cell 157 (1), 41-50. doi:10.1016/j.cell.2014.02.013

Schofield, R. (1978). The Relationship between the Spleen colony-forming Cell and the Haemopoietic Stem Cell. Blood Cells 4 (1-2), 7-25. vesicles (EVs) as communication tools between hematopoietic stem cells and the niche. Balise et al. focus on tetraspanins, a family of membrane scaffold proteins which compartmentalize membrane adhesion and signaling receptors as well as intracellular signaling molecules, providing a spatiotemporal organization of membrane-associated proteins to influence critical hematopoietic stem cell functions, such as quiescence, self-renewal, differentiation, adhesion, migration and signaling. Tetraspanins are also one of the most commonly found protein classes in EVs.

We will sign off with a sincere thank you to the reviewers who kindly devoted their time, in spite of the ongoing pandemic, to the rigorous vetting and enrichment of the submitted manuscripts. This topic would not have been possible without your effort.

\section{AUTHOR CONTRIBUTIONS}

$\mathrm{SM}$ wrote the draft of the editorial. $\mathrm{AB}$ and $\mathrm{MO}$ revised and approved the final version.

Conflict of Interest: The authors declare that the research was conducted in the absence of any commercial or financial relationships that could be construed as a potential conflict of interest.

Publisher's Note: All claims expressed in this article are solely those of the authors and do not necessarily represent those of their affiliated organizations, or those of the publisher, the editors and the reviewers. Any product that may be evaluated in this article, or claim that may be made by its manufacturer, is not guaranteed or endorsed by the publisher.

Copyright (c) 2021 Matsuura, Balduini and Onisto. This is an open-access article distributed under the terms of the Creative Commons Attribution License (CC BY). The use, distribution or reproduction in other forums is permitted, provided the original author(s) and the copyright owner(s) are credited and that the original publication in this journal is cited, in accordance with accepted academic practice. No use, distribution or reproduction is permitted which does not comply with these terms. 